\title{
Graphical Histogram Algorithm for Integrated- Circuit-Piezoelectric-Type Accelerometer for Health Condition Diagnosis and Monitoring
}

\author{
Chao-Chung Peng, ${ }^{*}$ Chih-Hsiang Kuo, and Chung-Yung $\mathrm{Wu}^{1}$ \\ Department of Aeronautics and Astronautics, National Cheng Kung University, Tainan, Taiwan \\ ${ }^{1}$ Automation and Instrumentation System Development Section, Iron and Steel Research and \\ Development Department, China Steel Corporation, Kaohsiung, Taiwan
}

(Received May 2, 2017; accepted September 29, 2017)

Keywords: IEPE-type sensor, signal processing, diagnosis and monitoring

In this study, a smart automatic health status diagnosis and monitoring scheme for an integrated-circuit-piezoelectric (IEPE)-type accelerometer is presented. In China Steel Corporation (CSC), IEPE-type accelerometers have been widely and frequently used for machine vibration measurement. Since a valuable monitoring report always counts on the precise measurement of IEPE-type accelerometers, the health condition of the sensors must be guaranteed. However, there are now more than two thousand IEPE accelerometers attached to field machines and some of them are not easy to reach. The point-by-point diagnosis of those sensors by field workers will require a large maintenance effort and is not efficient. As a result, in the pursuit of the socalled smart factory and the enhancement of the production process as well as attenuate numerous human maintenance efforts, a graphical histogram algorithm (GHA) health condition diagnosis and monitoring strategy is proposed. By the analysis of the histogram distribution and the use of spline interpolation on the IEPE accelerometer excitation signals, characteristic profiles can be extracted. Therefore, different health conditions should be classified systematically. Finally, the status of IEPE accelerometers can be automatically identified by estimating the correlations between the characteristic profiles. Experiments have been conducted to verify the feasibility of the proposed GHA.

\section{Introduction}

The Iron \& Steel Research \& Development Department (I\&S-R\&D) of China Steel Corporation (CSC) began its development of intelligent facility diagnosis methods in 2006. With the accumulation of critical sensing data from equipment and facilities under operation, the research department has implemented dozens of monitoring and predictive maintenance solutions on CSC's strip mills. The development outcomes are highly influential to the company's improvement of reducing operation cost, and to its aim of increasing production line reliability by implementing an intelligent diagnosis system. ${ }^{(1-4)}$

The CSC's R\&D department spent two years to fully develop its first online monitoring system, which was named "Facility Online Monitoring and Diagnosis System (FOMOS)", and began its

*Corresponding author: e-mail: ccpeng@mail.ncku.edu.tw

http://dx.doi.org/10.18494/SAM.2017.1738

ISSN 0914-4935 C MYU K.K. 
implementation on the production line in 2008. The core technique of the diagnosis method is to analyze the vibration signals gathered from the accelerometers attached to the equipment such as milling machines, ${ }^{(1)}$ bearings, ${ }^{(5)}$ high-speed motors, ${ }^{(6)}$ and certain electrohydraulic systems. ${ }^{(7)}$ This allows the real-time monitoring of the operation condition of the facility. FOMOS provides detailed insight into the causes of equipment malfunction and produces alerts and warnings, which are significantly helpful for onsite operators to take proper actions in a short response time. By implementing FOMOS, reducing downtime caused by unexpected equipment malfunction can be achieved, and this benefits the company by reducing lost profit and increasing the equipment lifetime.

FOMOS has been vastly implemented in CSC production lines, and more than two thousand integrated-circuit-piezoelectric (IEPE)-type ${ }^{(8)}$ accelerometers have been attached to equipment in the field. In facilities, most are under harsh environment with high temperature and high humidity; this makes the accelerometers prone to damage and, ultimately, malfunction. This indicates that, for the results from a diagnosis system to be trustworthy, ensuring that the accelerometers are in a healthy condition is a crucial first step to further ensure the signal reliability of the entire diagnosis system. If an abnormally operating accelerometer is not identified, this will further corrupt the diagnosis results produced by the monitoring and analysis system. Furthermore, the accelerometers are distributed around a large operation space, and thus conducting a field check by manpower is extremely inefficient; even if one check point only takes up 5 to $10 \mathrm{~min}$, the overall time consumption will be tremendous. While under a harsh environment, dispatching workers to perform an onsite check also increases the risk of the workers. Both putting employees in danger and increasing maintenance cost are not desirable options.

During the conduct of this research, it has been found that the characteristics of the excited signals acquired from accelerometers may vary from one another. Furthermore, the signal characteristics are highly dependent on data acquisition (DAQ) devices. In other words, if the DAQ hardware is replaced, the signal acquired from the new hardware set may vary from the original one. Thus, without the adaptability of the classification algorithm, the results of the classification may be different from the real situation.

In the literature, publications regarding this issue are rare. To more effectively solve the problem mentioned above, in this research, we proposed a health condition diagnosis method for an IEPE-type accelerometer. By measuring the AC/DC excited-signal dynamic characteristics of the accelerometer, and with an established mathematical model to learn to classify the results, the health condition of the accelerometer can be determined. At the same time, the problem of the dependence of the signal on the DAQ hardware is solved. With the aid of this technique in the field, the sensor status can be identified automatically. Therefore, local field technicians will be notified if any maintenance is required.

Last but not the least, in this research, we have proven the reliability of the technique after performing reliability tests with a thousand data sets, and the algorithm has already been implemented under the original FOMOS structure. The accelerometer diagnosis method provided by this research not only reduces the human resources and human risk to perform maintenance check on the conditions of accelerometers, but also realizes factory automation and self-diagnosis through the implementation of the intelligent diagnosis system. 


\section{Graphical Histogram Algorithm (GHA)}

According to reports on the condition of on-field accelerometers, the operating condition and their corresponding excited signal differences may be classified under five categories: (a) normal, (b) reverse connection, (c) open circuit, (d) short circuit, and (e) abnormal. For the response signals of the first four categories mentioned above, see also Fig. 1. If the signal characteristics are not categorized in the first four types, then they are classified as abnormal.

However, since the recorded response signals may vary when using different DAQ cards, the characteristics of the recorded response signals may be different if different DAQ hardware sets are used to record the same accelerometer. In other words, since the signal characteristics of the above-mentioned five categories are coupled with the characteristics of the DAQ hardware set used for their respective data recording, this may lead to wrong classification results. For example, Fig. 2 shows the AC/DC excited signal recording of the same normal-operating accelerometer. With the use of different DAQ hardware sets (e.g., NI-PXI DAQ and NI-Compact DAQ) for data collection, the signal dynamic characteristics vary. In addition, there are many other equipment that exist on the same site, causing interference and noises during measurement and recording, lowering the
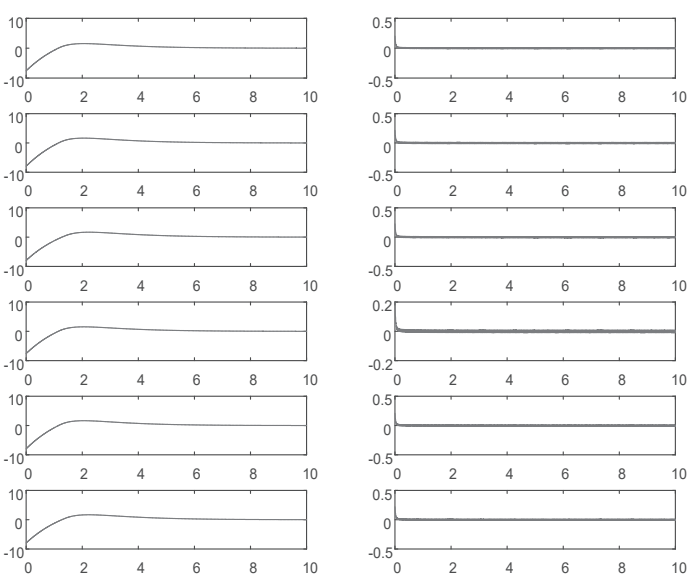

(a)
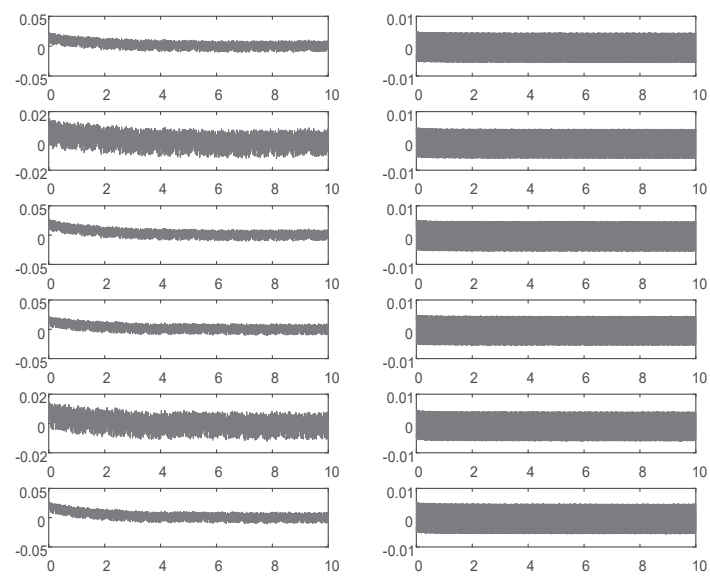

(c)
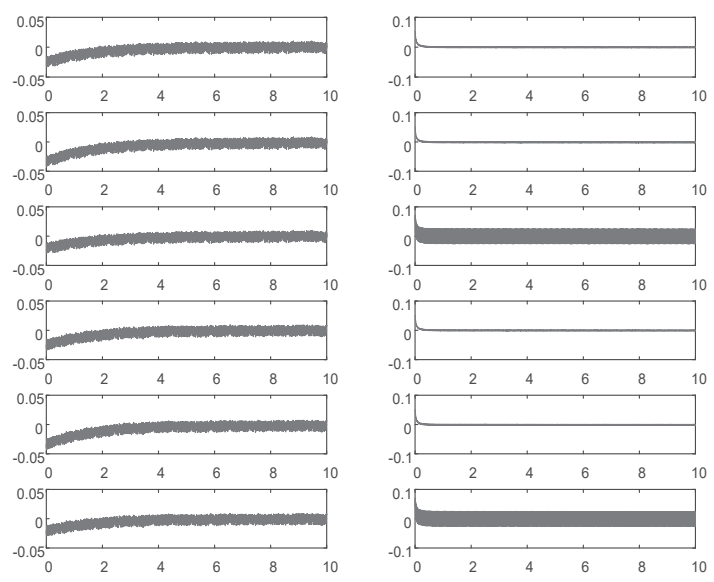

(b)
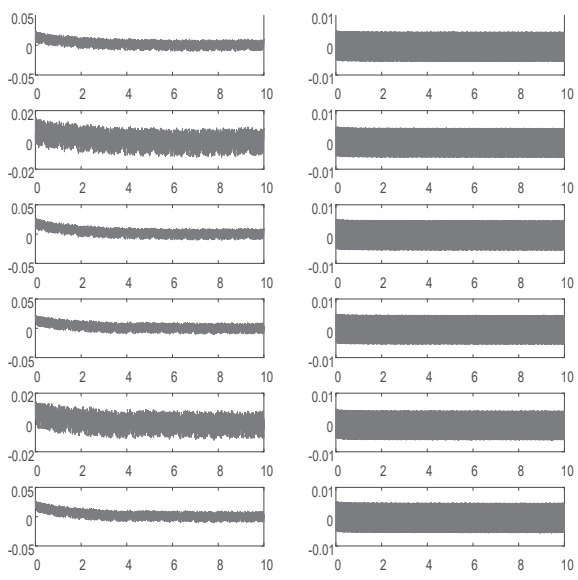

(d)

Fig. 1. IEPE-type accelerometer AC/DC excited signals under different conditions. (a) Normal, (b) reverse connection, (c) open circuit, and (d) short circuit. 

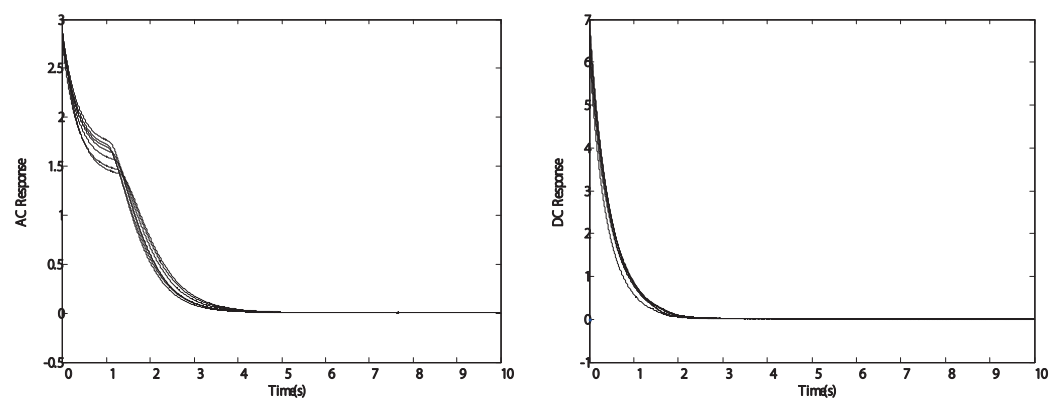

(a)
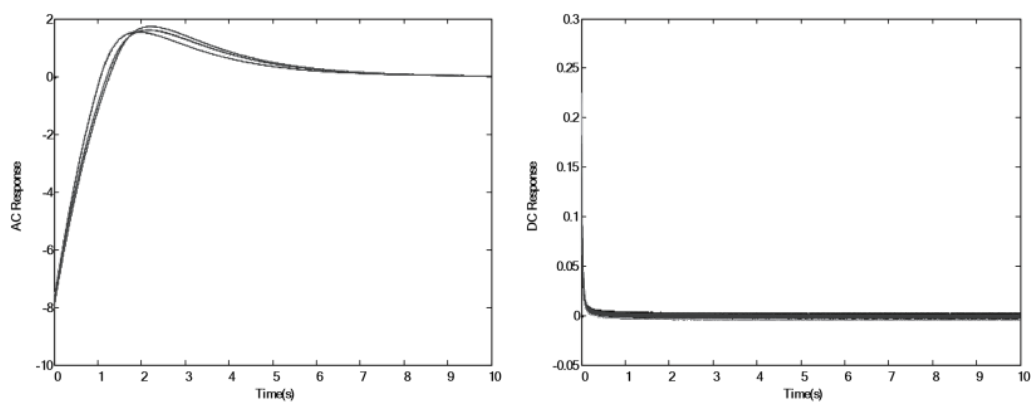

(b)

Fig. 2. AC/DC excited signals acquired from normal-operating multichannel accelerometer using different DAQ cards. Device: (a) NI-PXI DAQ and (b) NI-Compact DAQ.

accuracy of the classification results.

To tackle the above-mentioned challenge, in this research, we proposed a learning algorithm to automatically extract characteristics from excited signals, so as to solve the signal coupling problem and increase the flexibility of hardware arrangement and diagnosis robustness. By utilizing a signal preprocessing method, we can increase the stability of the acquired excited signals and significantly increase the reliability of the characteristic learning model. Lastly, the developed system automatically compares the correlation of the AC/DC excited signals with the trained model to finish the process of automatic classification and diagnosis.

\subsection{Signal prefilter design}

In most industrial applications, IEPE-type accelerometers are used in harsh environments, while being simultaneously exposed to interference caused by various surrounding equipment. Thus, during measurement, signal noises may be added to the records. Figure 1 shows the AC/DC excited signal acquired in a lab environment. However, measurements taken in a real industrial environment may be subject to differences caused by environment uncertainty. As a result, to construct a robust histogram, the uncertain noise must be analyzed and removed from the AC/DC excited signals. In the following, a finite impulse response (FIR) digital filter is considered.

Consider a linear time-invariant system with the impulse response $h[n]$; the output sequence $y[n]$ is related to the input sequence $u[n]$ through the convolution sum 


$$
\begin{aligned}
y[n] & =h[n] \times u[n]=\sum_{m=-\infty}^{\infty} h[m] u[n-m]=\sum_{m=-\infty}^{\infty} h[m] e^{j \omega(n-m)} \\
& =\left(\sum_{m=-\infty}^{\infty} h[m] e^{-j \omega m}\right) e^{j \omega n}=\left(\sum_{m=-\infty}^{\infty} h[m] e^{-j \omega m}\right) u[n] \\
& =H(j \omega) u[n]
\end{aligned}
$$

where $u[n], y[n]$, and $H(j \omega)$ are filter input, output, and frequency response, respectively.

Specify Eq. (1) using a moving average filter as follows:

$$
y_{\text {out }}(k)=\frac{1}{N} \sum_{i=0}^{N-1} u_{\text {in }}(k-i)
$$

From Eqs. (1) and (2), it follows that

$$
H(j \omega)=\sum_{m=-\infty}^{\infty} h[m] e^{-j \omega m}=\frac{1}{N} \sum_{m=0}^{\infty} e^{-j \omega m}=\frac{1}{N} \cdot \frac{1-e^{-j \omega N}}{1-e^{-j \omega}} .
$$

Note that the window size $N$ must be designed for a specific filtering purpose, which represents Eq. (3) by

$$
H(j \omega) \frac{1}{N} \cdot \frac{e^{-j \omega N / 2}}{e^{-j \omega / 2}} \cdot \frac{\sin (\omega N / 2)}{\sin (\omega / 2)}
$$

where the magnitude response is

$$
|H(j \omega)|=\frac{1}{N} \cdot\left|\frac{e^{-j \omega N / 2}}{e^{-j \omega / 2}} \cdot \frac{\sin \left(\frac{\omega N}{2}\right)}{\sin \left(\frac{\omega}{2}\right)}\right|=\frac{1}{N} \cdot\left|\frac{\sin \left(\frac{\omega N}{2}\right)}{\sin \left(\frac{\omega}{2}\right)}\right| .
$$

Equation (5) shows that $|H(j \omega)|=0$ when $\omega N=2 n \pi, n \in Z^{+}$. Equation (5) shows that, for a given $N$, specific multiband frequencies,

$$
\omega=\omega_{b}=2 n \pi / N
$$

are removed from raw signals, as illustrated in Fig. 3. For practical implementation, the window size $N$ is determined from the results of frequency spectrum analysis.

\subsection{Cubic spline for characteristic profile construction}

For different IEPE-type accelerometer profiles, time domain responses are represented by histograms. Since a histogram provides signal distribution features for different sensor conditions, the following is for constructing the characteristic profile using a cubic spline. ${ }^{(9,10)}$

For a given histogram, the tips of the bars can be connected by a general cubic spline defined as 

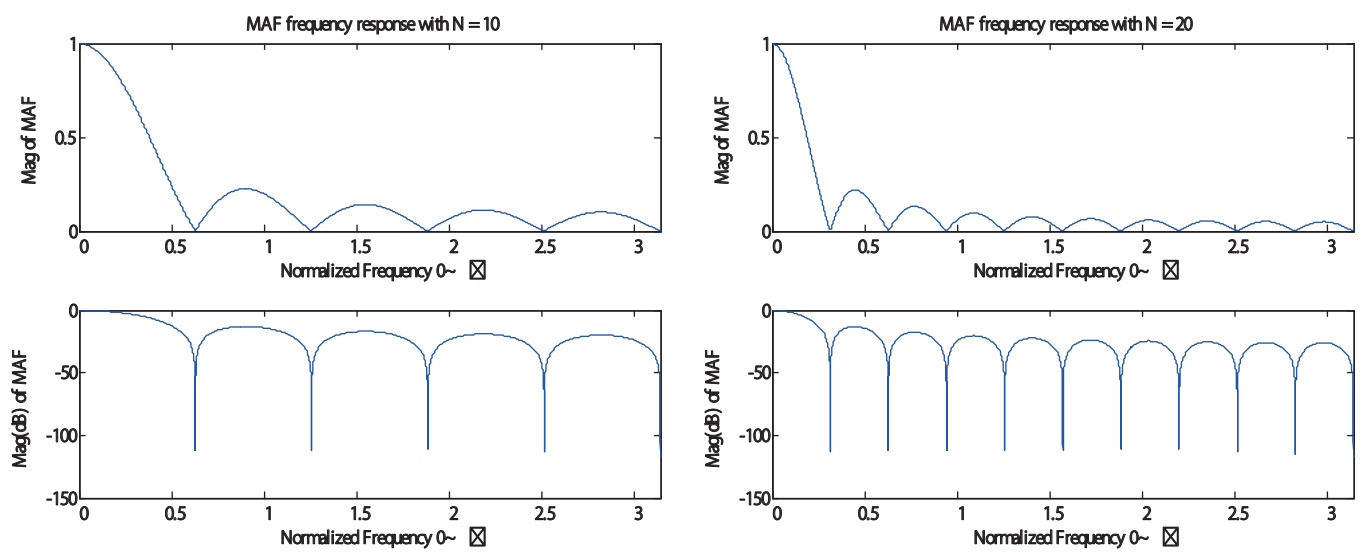

Fig. 3. (Color online) Frequency response of the signal prefilter.

$$
f_{i}=a_{i}+b_{i}\left(t-t_{i}\right)+c_{i}\left(t-t_{i}\right)^{2}+d_{i}\left(t-t_{i}\right)^{3},
$$

where $a_{i}=x_{i}$ and $i=1, \ldots, n$. The variables $a_{i}, b_{i}, c_{i}$, and $d_{i}$ are the profile coefficients and $\left(t_{i}, x_{i}\right)$ represents the information of bars from the given histogram.

To achieve a smooth and continuous characteristic profile, the following equations must be satisfied simultaneously.

$$
\begin{gathered}
x_{i}+b_{i} h_{i}+c_{i} h_{i}^{2}+d_{i} h_{i}^{3}=x_{i+1} \\
b_{i}+2 c_{i} h_{i}+3 d_{i} h_{i}^{2}=b_{i+1} \\
c_{i}+3 d_{i} h_{i}=c_{i+1}, h_{i}=t_{i+1}-t_{i}
\end{gathered}
$$

Considering that the 2nd derivatives at the first and last points are zero leads to $c_{1}=c_{n}=0$.

Let $d_{i}=\left(c_{i+1}-c_{i}\right) / 3 h_{i}$. Equations (8) and (9) can be represented by

$$
\begin{gathered}
x_{i}+b_{i} h_{i}+\frac{h_{i}^{2}}{3}\left(c_{i+1}+2 c_{i}\right)=x_{i+1}, \\
b_{i}+\left(c_{i+1}+c_{i}\right) h_{i}=b_{i+1},
\end{gathered}
$$

respectively. Equation (11) can be written as

$$
b_{i}=\frac{x_{i+1}-x_{i}}{h_{i}}-\frac{h_{i}}{3}\left(c_{i+1}+2 c_{i}\right) \text {. }
$$

Considering the index $i-1$ for Eqs. (12) and (13) gives

$$
b_{i-1}+\left(c_{i-1}+c_{i}\right) h_{i-1}=b_{i},
$$




$$
b_{i-1}=\frac{x_{i}-x_{i-1}}{h_{i-1}}-\frac{h_{i-1}}{3}\left(c_{i}+2 c_{i-1}\right) .
$$

Substituting Eqs. (13) and (14) into Eq. (15) gives

$$
c_{i-1} h_{i-1}+2\left(h_{i-1}+h_{i}\right) c_{i}+c_{i+1} h_{i}=3 \frac{x_{i+1}-x_{i}}{h_{i}}-3 \frac{x_{i}-x_{i-1}}{h_{i-1}} .
$$

Equation (16) can be written as a matrix from

$$
\left[\begin{array}{ccccc}
1 & 0 & \cdots & 0 & 0 \\
h_{1} & 2\left(h_{1}+h_{2}\right) & h_{2} & 0 & 0 \\
\vdots & \vdots & \ddots & \vdots & \vdots \\
0 & 0 & h_{n-2} & 2\left(h_{n-2}+h_{n-1}\right) & h_{n-1} \\
0 & 0 & \cdots & 0 & 1
\end{array}\right]\left[\begin{array}{c}
c_{1} \\
c_{2} \\
\vdots \\
c_{n-1} \\
c_{n}
\end{array}\right]=\left[\begin{array}{c}
0 \\
3\left(f\left[t_{3}, t_{2}\right]-f\left[t_{2}, t_{1}\right]\right) \\
\vdots \\
3\left(f\left[t_{n}, t_{n-1}\right]-f\left[t_{n-1}, t_{n-2}\right]\right) \\
0
\end{array}\right],(17)
$$

where $f\left[t_{i}, t_{i}\right]=\left(x_{i}-x_{j}\right) /\left(t_{i}-t_{j}\right)$.

\subsection{Characteristic profile correlation}

From the results described in Sects. 2.2 and 2.3, the characteristic profiles of AC/DC signals for all the IEPE-type accelerometer conditions (i.e., normal, abnormal, and so on) can be modeled by Eq. (7) subject to different coefficients.

As a result, for a given IEPE-type sensor to be measured, the health status can be detected by evaluating the correlation coefficient among all the characteristic profiles as follows:

$$
\eta=\frac{\operatorname{Cov}\left(y_{\text {model }}, y_{\text {sensor }}\right)}{\sigma_{\text {model }} \cdot \sigma_{\text {sensor }}}
$$

where $y_{\text {model }}$ and $y_{\text {sensor }}$ represent the characteristic profiles from the model set and new measurement, respectively. $\sigma_{\text {model }}$ and $\sigma_{\text {sensor }}$ are the corresponding deviations. In other words, for a given IEPE-type sensor, the characteristic profile is firstly determined and then the classification can be carried out by calculating the correlation coefficient between all the models.

The procedure of the IEPE-type sensor health monitoring and detection is summarized in Fig. 4. The automatic abnormality diagnosis technique proposed in this research can be separated into two main parts. The first part is shown in the left part of the flow chart, which includes feature extraction and characteristic profile construction. In order to establish the database, the first step is to collect datasets from accelerometers under different operation conditions. Through noise filtering, multiple histograms can be established. Next, using the cubic spline, one may extract the characteristic functions from the distribution results of the histograms. Lastly, these characteristic functions will be used as the AC/DC signal feature golden patterns. We may use such golden patterns to identify the unknown operation conditions of the accelerometer by comparing the unknown data with the established golden pattern.

In the second part of the process, which is shown in the right half of the flow chart, feature 


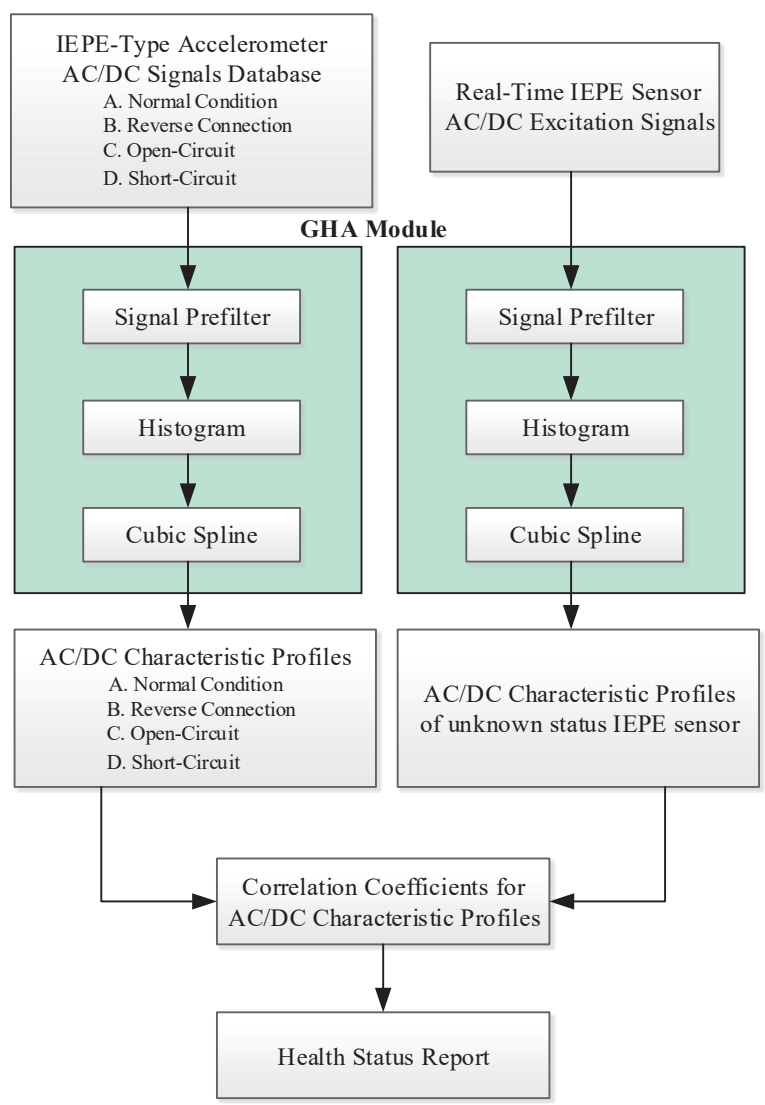

Fig. 4. (Color online) Automatic IEPE-type accelerometer health sensing procedure.

matching is performed. By performing the same steps from the first part to establish the GHA module of the signals from the unknown accelerometer, the characteristic function of the statusunknown accelerometer may be established. Then, a coefficient correlation analysis between the generated characteristics function with the golden pattern may be performed; this then finishes the diagnosis.

Through the process described above, the IEPE-type accelerometer automatic condition diagnosis algorithm can effectively differentiate the four different conditions mentioned above and can achieve accelerometer automatic abnormality diagnosis. Moreover, the algorithm adopts an independent learning method in order to provide more robust accelerometer condition diagnosis results, and the method also overcomes the challenge raised by the different electrical characteristics of the different electrical hardware sets used.

\section{Experiment}

In the experiment, a NI-DAQ system shown in Fig. 5 is applied as the measurement platform and the algorithm is realized by LABVIEW. To illustrate the feature of the prefilter used in the proposed GHA, DC signals subject to different levels of measurement noise are demonstrated.

Figure 6 illustrates DC excited signals of three normal sensors from a local field subject to clear surrounding AC current perturbations. According to the frequency spectrum, it is clear that 


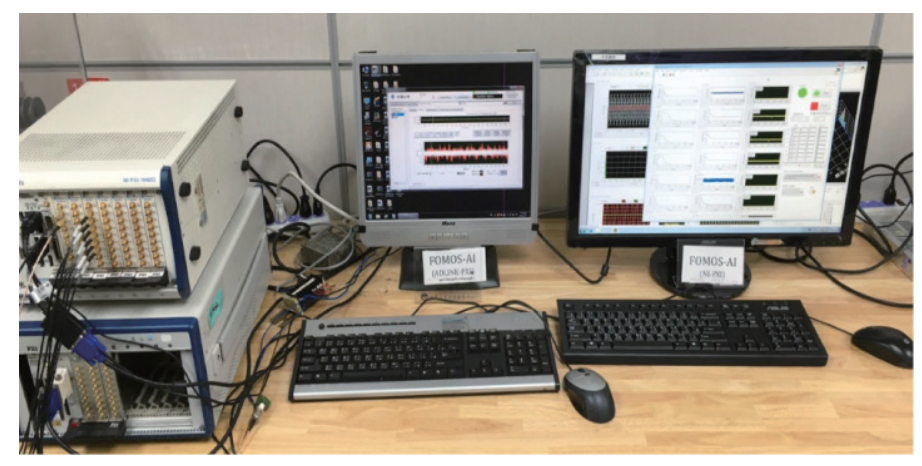

Fig. 5. (Color online) DAQ system setup.
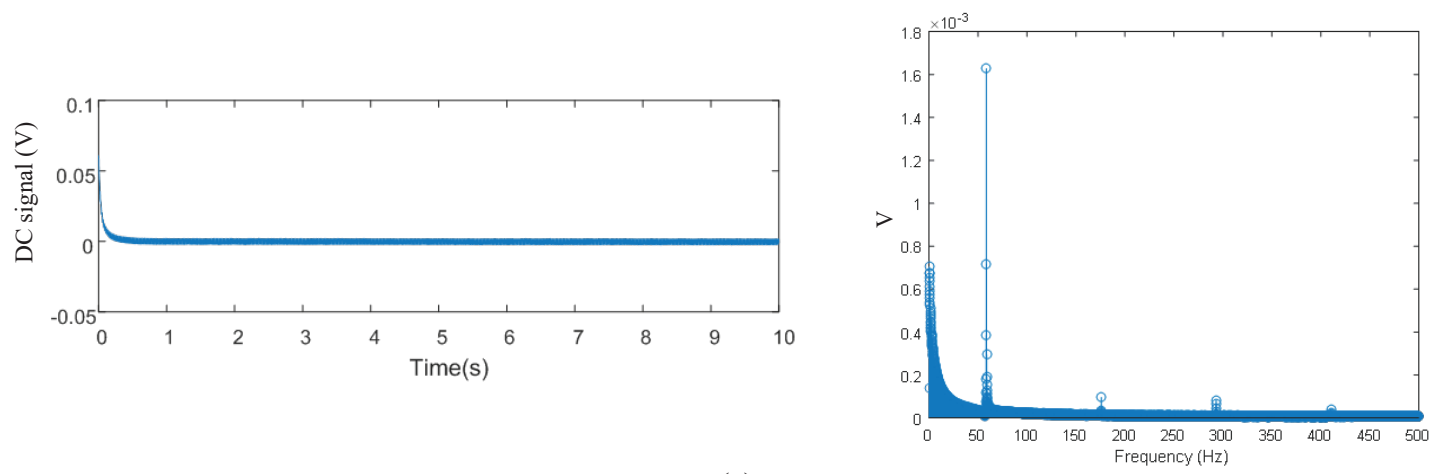

(a)
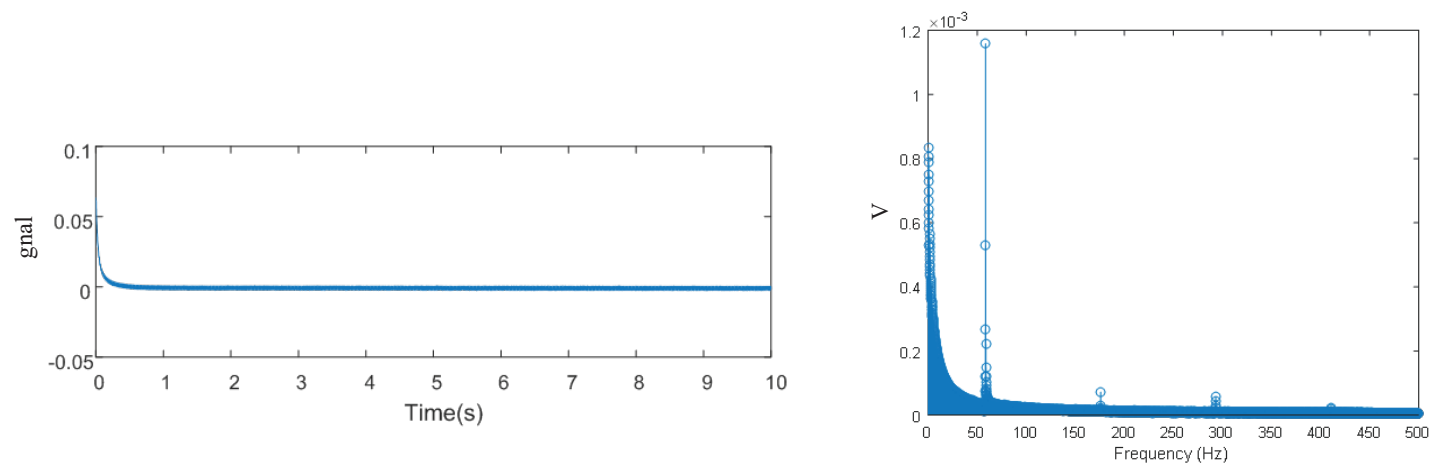

(b)
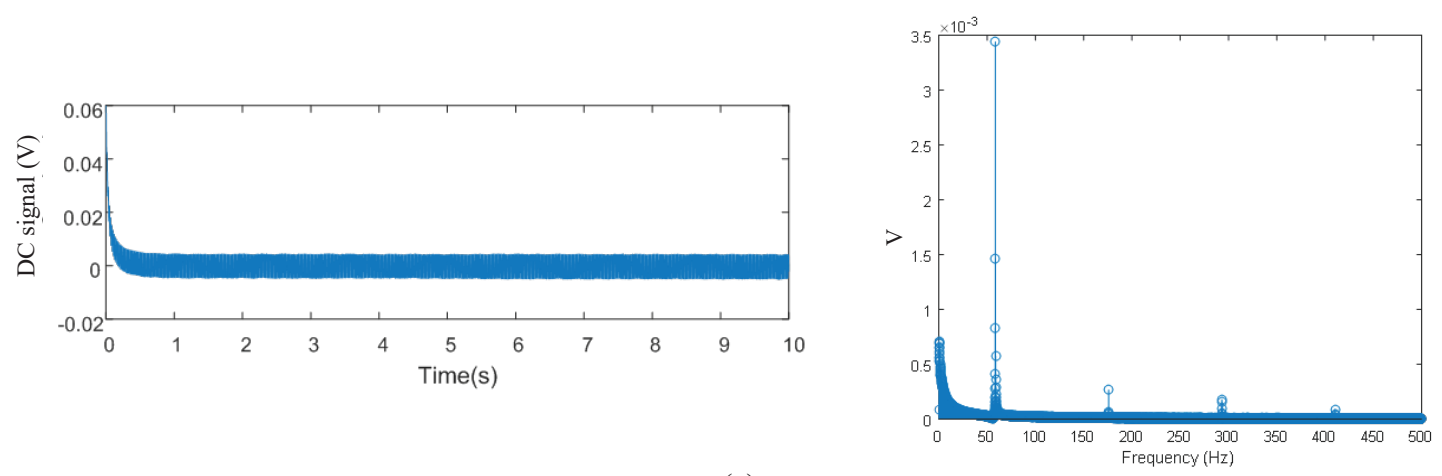

(c)

Fig. 6. (Color online) Excited DC signals under different noise environments. Time/frequency responses of (a) $\mathrm{CH}-1$, (b) $\mathrm{CH}-2$, and (c) $\mathrm{CH}-3$. 
their noise levels are different. The corresponding histograms are shown in Figs. 7(a)-7(c), which illustrate less feature consistency. From the noise distribution, noise peaks are observed 59, 177, 295 , and $413 \mathrm{~Hz}$. Hence, for the given sampling rate of $1 \mathrm{kHz}$, the window size for the prefilter is set to be 17, which results in Figs. 7(d)-7(f). It is evident that, by applying the prefilter, the robustness of the histogram can be significantly improved.

On the basis of the given data of a few sensors, the model for each health status can be constructed. Figure 8 illustrates the averaged histograms for the excited AC/DC signals collected from normal IEPE sensors, where the red lines represent the corresponding characteristic profiles. Once the models are completed, the final step is to evaluate the correlation coefficients.

Tables 1 and 2 show the correlation evaluation results of $\eta_{A C}$ and $\eta_{D C}$, respectively. These tables clearly show that the correlation coefficients are mostly higher than 0.9 if the sensor conditions are matched. However, since certain mismatched sensor conditions could also return $\eta>0.9$, decision making must consider $\eta_{A C}$ and $\eta_{D C}$ simultaneously. In other words, the final health detection result is made by using $\eta=\eta_{A C} \times \eta_{D C}$ and the maximum $\eta$ returns the diagnosis class.

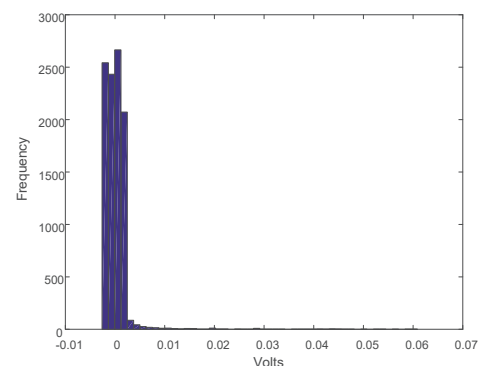

(a)

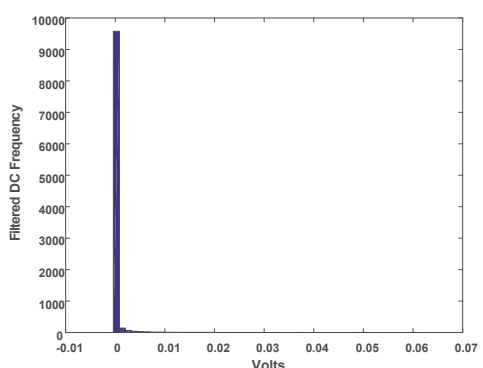

(d)

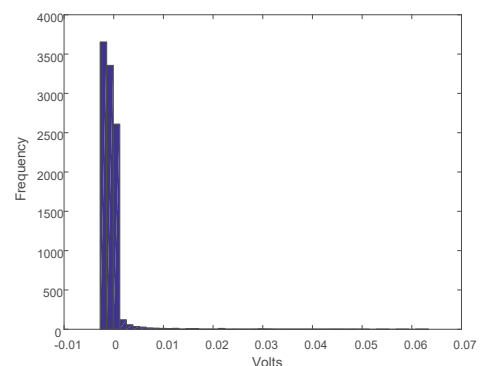

(b)

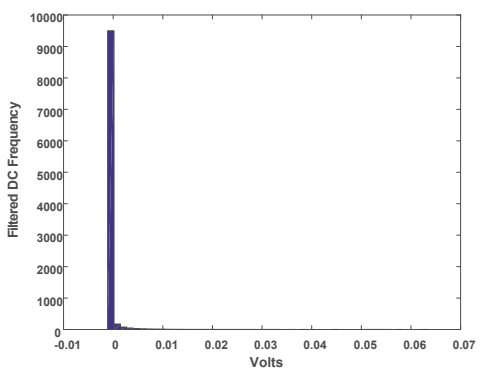

(e)

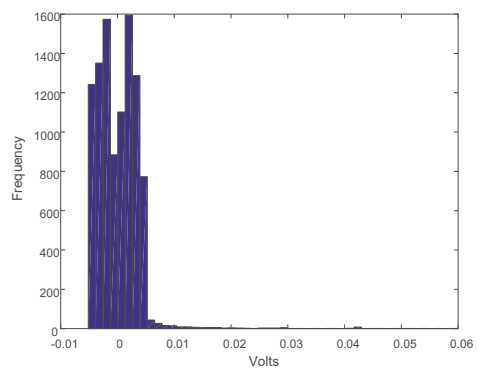

(c)

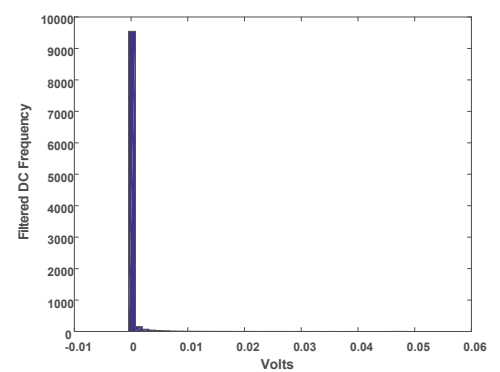

(f)

Fig. 7. (Color online) Histograms of DC excited signals under different noise environments: (a)-(c) without and (d)(f) with prefilter.

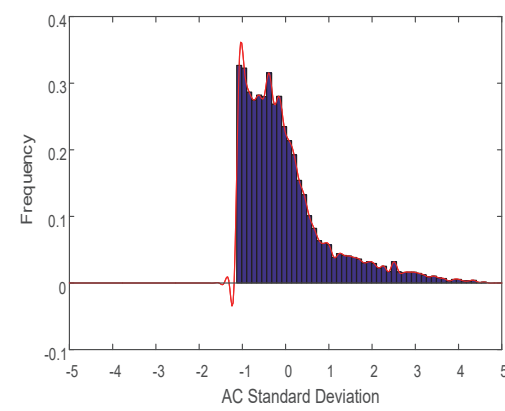

(a)

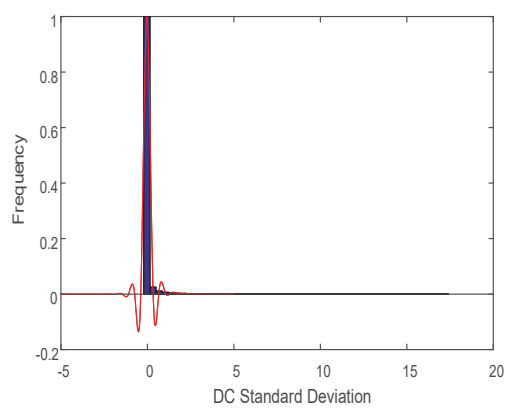

(b)

Fig. 8. (Color online) Histograms of AD/DC excited signals and characteristic profiles of normal sensors. 
Table 1

Correlation evaluation results of AC signal.

\begin{tabular}{lcccc}
\hline$\eta_{A C}$ & Reverse & Normal & Short-circuit & Open-circuit \\
\hline Ref reverse & $>0.9$ & $0.5-0.65$ & $<0.7$ & $>0.8$ \\
Ref normal & $0.6-0.7$ & $>0.9$ & $0.5-0.9$ & $<0.6$ \\
Ref short-circuit & $<0.7$ & $0.63-0.9$ & $>0.9$ & $<0.6$ \\
Ref open-circuit & $>0.8$ & $<0.6$ & $0.5-0.7$ & $>0.9$ \\
\hline
\end{tabular}

Table 2

Correlation evaluation results of DC signal.

\begin{tabular}{lcccc}
\hline$\eta_{D C}$ & Reverse & Normal & Short-circuit & Open-circuit \\
\hline Ref reverse & $>0.94$ & $<0.95$ & $<0.5$ & $0.4-0.8$ \\
Ref normal & $0.77-0.95$ & $>0.98$ & $<0.55$ & $0.4-0.8$ \\
Ref short-circuit & $<0.5$ & $<0.6$ & $>0.98$ & $0.7-0.98$ \\
Ref open-circuit & $<0.65$ & $<0.7$ & $0.9-0.95$ & $>0.89$ \\
\hline
\end{tabular}

\section{Conclusions}

In this research, an automatic health condition diagnosis method for an IEPE-type accelerometer, which is widely used in CSC facilities, is developed. By acquiring the AC/DC coupling charge and discharge dynamic response signals from the accelerometer, the developed algorithm then uses the cubic spline to compute the models corresponding to the categories. The trained model can then be used for accelerometer automatic abnormality diagnosis. While using this proposed diagnosis method, the excited signals acquired from the tested accelerometers will be compared with the trained golden patterns. Then, the diagnosis results will be sent to technicians immediately, while providing suggestions on the condition of the abnormally operating accelerometers. The proposed algorithm has been successfully integrated into the recent CSCFOMOS, increasing the completeness and performance of the vibration diagnosis function. After on-field testing, the method is proved to perform robust accelerometer automatic health condition diagnosis. Also, the method provides technicians with suggestions on the abnormality category. This results in significant reductions in time and human resources needed to perform health check on accelerometers. At the same time, through programmable software, the method enables CSC facilities to realize fully automatic self-monitoring and diagnosis of the accelerometer health condition. Finally, experiments are performed to verify the feasibility of the proposed GHA.

\section{Acknowledgments}

The work was partially supported by the Ministry of Science and Technology, Taiwan, under grant Nos. MOST 106-2218-E-006-004 and MOST 106-2221-E-006-138.

\section{References}

1 C. C. Wang, C. H. Ko, J. S. Shiau, and W. Long: IEEE Int. Symp. Optomechatronic Technologies (IEEE, 2014) p. 105.

2 J. S. Shiau and C. C. Wang: China Steel Technical Report (2012) No. 327.

3 H. Honiyo and H. Watanabe: Iron. Steel Eng. May (1975) 31. 
4 C. C. Wang: Conf. South East Asia Iron and Steel Institute (Bangkok, 2008).

5 T. C. Liu and T. Y. Wu: Smart Sci. 5 (2017) 150.

6 C. C. Hwang, C. J. Hong, and C. Y. Chang: Smart Sci. 5 (2017) 21.

7 H. M. Chen, G. W. Yang, and C. C. Liao: Smart Sci. 2 (2014) 132.

8 Measuring Vibration with Accelerometers: http:/www.ni.com/white-paper/3807/en/

9 S. C. Chapra: Applied Numerical Method with MATLAB, 3rd ed. (McGraw-Hill, New York, 2012) p. 439.

10 C. S. Lin, P. R. Chang, and J. Y.S. Luh: IEEE Trans. Autom. Control 14 (1983) 1066.

\section{About the Authors}

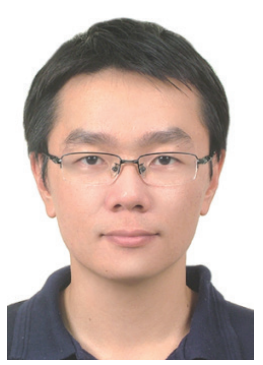

Chao-Chung Peng received his B.S. degree from the Department of Aeronautics and Astronautics, National Cheng Kung University, in 2003, Tainan, Taiwan. After his first year as an M.S. degree student in the same department, he was promoted to be a Ph.D. candidate. He started his study under the Ph.D. program in 2004 and received his Ph.D. degree in 2009. From 2008 to 2009, he was with the Department of Engineering, Leicester University, United Kingdom, for his advanced research. In 2009, he was awarded a membership in the Phi Tau Phi Scholastic Honor Society. After one year of military service in Kaohsiung, he worked in the Department of Mechanical Engineering, National Cheng Kung University, as a postdoctoral fellow from 2010 to 2012. From the end of 2012, he moved to ADLINK Technology and served as a Senior Engineer in the Embedded System Development Section, Measurement and Automation Department, Taipei, Taiwan. His research mainly included system modeling/identification, robust controller design, and embedded system integration. From 2014 to 2016, he joined the Automation \& Instrumentation System Development Section, Iron \& Steel Research \& Development Department, China Steel Corporation (CSC), Kaohsiung, Taiwan. His major works included the analysis/control of mill system and mechanical system online monitoring and fault diagnosis. He is currently an Assistant Professor of the DAA, NCKU. His research interests include linear/nonlinear control, motion control, robotics, sliding mode control, unmanned vehicle design, and embedded system integration.

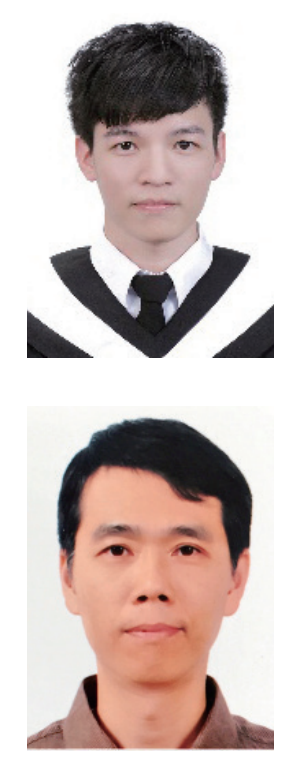

Chih-Hsiang Kuo received his B.S and the M.S. degrees from the Department of Aeronautics and Astronautics, National Cheng Kung University, Taiwan, in 2015 and 2017, respectively. From 2015 to 2017, he was a concurrent research assistant at National Cheng Kung University, Taiwan.

Chung-Yung Wu earned his Ph.D. in mechanical engineering from Pennsylvania State University in 1998. He is currently a research scientist with the China Steel Corporation. His research interests are in the areas of machinery diagnosis, dynamic system modeling and analysis, and acoustics. 